\title{
Estilos de aprendizaje en la carrera de ingeniería en agropecuaria
}

\author{
Aimé Rosario Batista-Casacó \\ aimebatista@gmail.com \\ 0000-0002-1039-7414 \\ Facultad de Ciencias Agropecuarias, \\ Universidad Técnica Estatal de Quevedo. Ecuador. \\ Alexis Mendoza-Zambrano \\ alexismendozazambrano@gmail.com \\ 0000-0002-4747-5033 \\ Ministerio de Educación Quevedo - Los Ríos. Ecuador \\ Ana Ruth Álvarez-Sánchez \\ dra.arasanchez@gmail.com \\ 0000-0003-2780-8600 \\ Facultad de Ciencias Agropecuarias, \\ Universidad Técnica Estatal de Quevedo. Ecuador. \\ Mariuska Simón Fosten \\ mariuska.simon@reduc.edu.cu \\ 0000-0001-5502-8770 \\ Universidad de Camagüey "Ignacio Agramonte y Loynaz" \\ Cuba.

\section{Marlon Fernando Monge Freile mmongef@uteq.edu.ec 0000-0001-5397-910X} \\ Facultad de Ciencias Agropecuarias, \\ Universidad Técnica Estatal de Quevedo. Ecuador. \\ Mariasol Belén Culcay Véliz \\ mculcay@uteq.edu.ec \\ https://orcid.org/0000-0002-9330-8826 \\ Facultad de Ciencias Agropecuarias, \\ Universidad Técnica Estatal de Quevedo. Ecuador.

\section{Wilver Humberto Santana Alvarado \\ wsantanaa@uteq.edu.ec $\underline{0000-0002-6735-1257}$} \\ Facultad de Ciencias Agropecuarias, \\ Universidad Técnica Estatal de Quevedo. Ecuador.
}




\section{RESUMEN}

El objetivo del presente estudio fue, identificar los estilos de aprendizaje preferidos por los estudiantes de la carrera de ingeniería Agropecuaria, para garantizar su proceso de enseñanza aprendizaje, que sirva como base para diseñar estrategias didácticas y metodológicas futuras por parte de los docentes. Fueron seleccionados los estudiantes del segundo curso, como preámbulo de la asignatura (Sanidad Animal), primera materia práctica con animales vivos en el currículo de esta carrera. La información que se recolectó, fue almacenada en excell, en el que archivo que genera el propio inventario a través de un Formulario de Google. Fueron eliminados los atributos relacionados con fecha, hora, modalidad, por ser irrelevantes para lograr el objetivo trazado. Se empleó el programa estadístico SPSS 25.0 para Windows. La validez del Inventario de FelderSilverman se analizó a través de la aplicación de un análisis factorial exploratorio con rotaciones Varimax y para la confiabilidad de las diferentes dimensiones del test se aplicó la prueba de Cronbach. Se concluyó que en los estudiantes encuestados existía un equilibro entre los distintos aprendizajes propuestos por este inventario, aunque se pudo apreciar una tendencia hacia el aprendizaje activo, sensorial y visual, Los estilos secuencial y global, estuvieron completamente equilibrados.

Palabras clave: sanidad animal; proceso de enseñanza aprendizaje; estilos de aprendizaje 


\title{
Learning styles in the agricultural engineering career
}

\begin{abstract}
The objective of this study was to identify the learning styles preferred by students of the Agricultural Engineering career, to guarantee their teaching-learning process, which serves as a basis for designing future teaching and methodological strategies by teachers. The second year students were selected as a preamble to the subject (Animal Health), the first practical subject with live animals in the curriculum of this career. The information that was collected was stored in excell, in which a file that generates the inventory itself through a Google Form. Attributes related to date, time, and modality were eliminated as they were irrelevant to achieve the objective set. The statistical program SPSS 25.0 for Windows was used. The validity of the Felder-Silverman Inventory was analyzed through the application of an exploratory factor analysis with Varimax rotations and the Cronbach test was applied for the reliability of the different dimensions of the test. It was concluded that in the surveyed students there was a balance between the different learning proposed by this inventory, although a trend towards active, sensory and visual learning could be appreciated. The sequential and global styles were completely balanced.
\end{abstract}

Keywords: Animal Health, teaching-learning process, learning styles

Artículo recibido: 05 octubre. 2021 Aceptado para publicación: 02 noviembre 2021

Correspondencia: aimebatista@gmail.com Conflictos de Interés: Ninguna que declarar 


\section{INTRODUCCIÓN}

El aprendizaje desarrollador dentro de la educación surge en la década del 80, autores como Keefe y Monk (1986) estuvieron entre los primeros en hacer alusión al tema. Este se encuentra estrechamente vinculado con los diferentes estilos de aprendizajes, relacionados con la forma en que los individuos procesan la información, sienten y actúan en las diferentes situaciones de aprender, las cuales estarán sujetas al contexto donde este se desarrolla y de cómo procesan la información, basados en rasgos cognitivos, afectivos y fisiológicos; indicadores que muestran hasta cierto punto, como los estudiantes pueden percibir e interrelacionarse, en los diferentes ambientes estudiantiles, los cuales se revertirán ponderadamente en las diferentes actividades de aprendizaje, tanto prácticos como teóricos (García Gajardo et al., 2015).

Según Castellanos Simons (2001), este tipo de aprendizaje, es aquel, donde el individuo es capaz de apropiarse de forma creadora y activa del conocimiento, lo que contribuye al perfeccionamiento personal constante y al desarrollo de su autodeterminación, con mejor autonomía, relacionado íntimamente con los procesos de socialización y responsabilidad, con un marcado compromiso social.

Constituye un reto para el docente, actuar en un ambiente pedagógico donde se encuentran niveles desiguales de desarrollo intelectual, biológico y físico, con estilos de aprendizajes muy diferentes, donde priman motivaciones e intereses particulares, con ambientes socioculturales también diferentes, que indiscutiblemente repercuten en el proceso, por tanto, el docente se convierte en un artífice desde la diversidad, siempre y cuando mire el proceso pedagógico desde la óptica afectiva, cognitiva, motivacional y psicosocial (Torrano et al, 2017). Por lo que resulta indispensable que este conozca previamente los estilos de aprendizajes de sus estudiantes, en función de poder desarrollar recursos didácticos a la hora de impartir el contenido que permitan atender las características interindividuales de sus educandos, ya que las mismas van sujetas a las demandas educativas las cuales precisan ser contextualizadas, a los diferentes entornos donde estos se desarrollan (Baldozzi, 2015).

Las instituciones de educación superior, presentan un gran desafío para lograr un proceso de enseñanza aprendizaje motivador e innovador, de modo que a la hora de confeccionar los contenidos de las asignaturas del currículo, tienen el cometido de valorar todos los 
recursos didácticos necesarios para el proceso en estrecha relación con la manera en que aprenden sus estudiantes (Leal Bolaño et al, 2019).

Las carreras de ingeniería Agropecuaria se adaptan a las concepciones contemporáneas relacionadas con la pedagogía actual, que se basan en un aprendizaje desarrollador y formativo, donde el estudiante aprende a aprender, desempeñando un papel protagónico en su aprendizaje, contrario a las metodologías convencionales y clásicas donde solo el docente tenía un papel activo. Los aspectos que sirven como criterio primario para la valoración histórico - lógica de esta carrera se sustentan sobre la base de que los estudiantes que entran a ella, muchas veces no se encuentran completamente motivados con la elección, debido a una baja orientación profesional, o están estimulados por familiares que se desarrollan en labores agropecuarias con conocimientos muy empíricos, por lo que se les dificulta la asimilación de los contenidos, y resulta imprescindible que estos últimos sean científicos, motivadores y con carácter diferenciador para que puedan ser mejor asimilados, por lo que estas dificultades, deben ser resueltas con un currículo sólido, tal y como plantea Altuna Urdin et al, (2021), basado en un sistema de conocimientos que responda a las necesidades de la producción agropecuaria actual, a partir de un aprendizaje desarrollador y sostenible que permita el propio desempeño de los estudiantes, durante sus años de estudio (Batista Casacó et al, 2019) .

La Sanidad Animal, es una de las asignaturas que reciben los estudiantes en esta carrera, y se encarga de la prevención de enfermedades, así como el tratamiento, la erradicación y control de estas, ya sean exóticas o endémicas, siendo uno de los pilares en la seguridad alimentaria, al garantizar el abasto constante y suficiente de alimentos sanos y nutritivos para la población (Foy, 2017). Es preciso señalar que esta asignatura, es la primera de carácter práctico en el terreno donde se desarrollan las diferentes especies productivas, o sea, constituye el primer contacto físico de los estudiantes con los animales, por lo que es imprescindible, que el aspecto psicológico, vaya de la mano, con el motivacional y el pedagógico, para que el aprendizaje pueda ser desarrollador en el tiempo y modo real de cada uno de ellos.

Según la OIE, (2010), las noxas de origen animal, son responsables del $20 \%$ de las pérdidas en estas producciones, por lo que el dominio de los contenidos de esta asignatura para el futuro ingeniero zootecnista, es vital, en tanto, su acción apropiada y oportuna, puede prevenir animales enfermos, a través de un diagnóstico certero, extensivo a la 
prevención de enfermedades zoonóticas, que cada año, causan más afectaciones no solo a la salud animal, sino también a la humana.

Aunque los estilos de aprendizajes es un tema muy polémico, los autores del presente trabajo, determinaron emplear las cuatro dimensiones propuestas por Felder y Silverman (1988), descritas por Silva Sprok (2018), ya que este modelo, describen al estudiante en un ambiente pedagógico estructurado, capaz de receptar y procesar la información de una manera adecuada, en dependencia de sus preferencias a la hora de aprender; y al docente, le permite diseñar recursos didácticos de enseñanza aprendizaje motivadores en dependencia de los estilos de aprendizajes que predominan en los estudiantes (Juárez Lugo et al, 2012).

Dentro de las cuatro dimensiones en las que se enmarcan los estilos de aprendizajes se pueden encontrar: estudiantes sensoriales o intuitivos, estudiantes visuales o verbales, estudiantes activos o reflexivos, estudiantes secuenciales o globales.

- Los estudiantes sensoriales o intuitivos (percepción), tienden a percibir el mundo y aunque los estudiantes utilizan ambas facultades, la inmensa mayoría tiende a desenvolverse más en una u en otra. Los sensoriales usan sus órganos de los sentidos para observar y recopilar datos, son excelentes en describir hechos, en experimentar, resolver problemas a través de métodos estándares, y no les gustan las sorpresas, son detallistas, pacientes, memoristas, no les agradan las situaciones complicadas, aunque son cuidadosos y lentos en la realización de sus actividades. Los intuitivos, son más teóricos, inclinándose hacia los principios y la innovación. Aunque no les gusta la repetición, son muy buenos captando conceptos, y completas los deberes de forma rápida, por lo que en ocasiones son descuidados.

- Los estudiantes visuales o verbales (representación), reciben información de una forma específica. Se dividen en: a) visuales (vistas, cuadros, diagramas, símbolos); b) verbales (sonidos y palabras); y c) cenestésicos (gusto, el tacto y el olfato). Los visuales se relaciona directamente con los procesos de aprendizaje al percibir la información y con el gusto, la percepción, el tacto, el olfato y la manera en que estos individuos procesan la información. Los verbales tienen buena memoria para que lo que oyen y escuchan para luego repetir, recuerdan bien las discusiones, prefieren la explicación verbal y son buenos explicándoles cualquier situación a los demás. 
- Los estudiantes activos o reflexivos (procesamiento) experimentación, no les gustan las clases o actividades docentes que muestren información de forma pasiva, trabajan y aprenden mejor cuando se desenvuelven en grupos de trabajo y en actividades experimentales. Los reflexivos precisan de situaciones que les permitan pensar en la información que recibe. Trabajan mejor solos o con otra persona. Son propensos a idear teorías.

- Los estudiantes secuenciales o globales (comprensión) tienden a aceptar las actividades docentes tal y como el profesor se las presenta sintiéndose cómodos. Son lineales en la resolución de problemas. Son muy buenos en el análisis y pensamiento convergente, y aprenden mejor, cuando los docentes exponen materiales en una progresión constante de complejidad. Los globales presentan un aprendizaje fragmentado, se sienten perdidos por días o semanas y de repente hacen las cosas; a partir de allí, son capaces de entender el material, suficientemente bien, que dejan al resto de los compañeros desconcertados. Son divergentes de pensamiento y síntesis, tendiendo a moverse a realizar tareas con mayor grado de dificultad.

- Por lo que el objetivo de la presente investigación fue identificar los estilos de aprendizaje de los estudiantes que recibirán la asignatura Sanidad Animal, para garantizar el proceso de enseñanza aprendizaje en la misma, y que sirva de base para diseñar estrategias didácticas y metodológicas futuras por parte de los docentes de esta asignatura.

\section{MATERIALES Y MÉTODOS}

La investigación se desarrolló bajo muestreo no probabilístico a 53 estudiantes del segundo curso de la carrera de ingeniería en Agropecuaria, de la Universidad Técnica Estatal de Quevedo, en Los Ríos, Ecuador. Del total de estudiantes encuestados 33 eran hombre y 20 mujeres, con una edad promedio de 20 años.

Se empleó el instrumento de Felder-Silverma (1988), el cual está compuesto por 44 preguntas, con dos alternativas de respuestas. Este inventario lleva implícito 4 dimensiones: activo-reflexivo, sensitivo-intuitivo, visual-verbal y secuencial-global). Puntajes entre $-3 y+3$ en cada dimensión sugieren un estilo de aprendizaje equilibrado entre los dos polos de la escala, puntajes entre $-7 \mathrm{y}-5$ indican preferencia moderada hacia el estilo activo, sensitivo, visual o secuencial, puntajes entre $+5 \mathrm{y}+7$ indican 
preferencia moderada hacia el estilo reflexivo, intuitivo, verbal o global, finalmente, puntajes entre -11 y -9 indican una fuerte preferencia hacia el estilo activo, sensitivo, visual o secuencial, y puntajes entre +9 y +11 indican fuerte preferencia hacia el estilo reflexivo, intuitivo, verbal o global y puntajes entre +9 y +11 indican fuerte preferencia hacia el estilo reflexivo, intuitivo, verbal o global (Gómez et al, 2021).

Los estudiantes tuvieron la opción de participar o no en la encuesta (inventario) y la misma fue realizada con su consentimiento. El tiempo de recolección de los datos fue de 10 a 25 minutos.

La validez y confiabilidad del Inventario de Felder-Silverman fueron analizadas a través de análisis factorial exploratorio con rotaciones Varimax y la prueba de Cronbach respectivamente.

\section{RESULTADOS Y DISCUSIÓN}

La dimensión Activa - Reflexiva del inventario de Felder- Silverman (1988) aplicado a los estudiantes, mostró que el 60, 4 \% presentan un equilibrio entre ambas, con una fuerte preferencia hacia el aprendizaje activo (Figura 1), en correspondencia con Alonso et al (2007) y Tapias et al. (2012), lo que favorece que los mismos puedan ser motivados hacia el componente práctico tan importante en la asignatura Sanidad Animal, el cual está destinado a la garantía sanitaria de las diferentes especies de animales y a la vigilancia epidemiológica constante, sustentada en la observación clínica exhaustiva del perfil de las diferentes enfermedades en una población, ya sea animal o humana relacionadas con las condiciones de manejo zoonóticas, esto podrá desarrollarse a través de actividades docentes individuales o grupales que favorezcan lo investigativo, relacionado con los disímiles problemas cotidianos de la salud, de acuerdo con Pérez Matamoros, (2021), en este caso vinculado con los animales sobre el terreno práctico o lugares de crianza animal.

\section{Tabla 1}


Resultado general de los Estudiantes Encuestados con el Inventario Ferder-Silverman

\begin{tabular}{|c|c|c|c|c|c|c|c|c|c|c|c|}
\hline \multicolumn{3}{|c|}{ Activo-Relexivd } & \multicolumn{3}{|c|}{ lensorial-Intuitive } & & \multicolumn{2}{|c|}{ Visual-Verbal } & \multicolumn{3}{|c|}{ pecuencial-Globa } \\
\hline Pregunta No & A & B & Pregunta No. & A & B & Pregunta No. & A & B & Pregunta No & A & B \\
\hline 1 & & 1 & 2 & 1 & & 3 & & 1 & 4 & & 1 \\
\hline 5 & & 1 & 6 & & 1 & 7 & 1 & & 8 & & 1 \\
\hline 9 & 1 & & 10 & 1 & & 11 & 1 & & 12 & & 1 \\
\hline 13 & & 1 & 14 & & 1 & 15 & & 1 & 16 & & 1 \\
\hline 17 & & 1 & 18 & 1 & & 19 & 1 & & 20 & 1 & \\
\hline 21 & 1 & & 22 & & 1 & 23 & 1 & & 24 & & 1 \\
\hline 25 & 1 & & 26 & & 1 & 27 & 1 & & 28 & & 1 \\
\hline 29 & 1 & & 30 & & 1 & 31 & 1 & & 32 & & 1 \\
\hline 33 & & 1 & 34 & & 1 & 35 & 1 & & 36 & 1 & \\
\hline 37 & & 1 & 38 & 1 & & 39 & 1 & & 40 & 1 & \\
\hline 41 & 1 & & 42 & 1 & & 43 & 1 & & 44 & 1 & \\
\hline $\begin{array}{l}\text { Total } \\
\text { columna }\end{array}$ & 5 & 6 & & 5 & 6 & & 9 & 2 & & 4 & 7 \\
\hline $\begin{array}{l}\text { Resta } \\
\text { Menor al } \\
\text { mayor }\end{array}$ & & 1 & & 1 & & & 7 & & & 3 & \\
\hline \begin{tabular}{|l|} 
Asignar \\
Letra mayor
\end{tabular} & & B & & E & & & A & & & B & \\
\hline
\end{tabular}

Tabla 2

Estilos de Aprendizaje Desde el Perfil de un estudiante Encuestado con el Inventario Ferder-Silverman (1988.

\begin{tabular}{|l|l|l|l|l|l|l|l|l|l|l|l|l|l|}
\hline & 11 & 9 & 7 & 5 & 3 & 1 & 1 & 3 & 5 & 7 & 9 & 11 & \\
\hline ACTIVO & & & & & & & $\mathrm{X}$ & & & & & & REFLEXIVO \\
\hline SENSORIAL & & & & & & & $\mathrm{X}$ & & & & & & INTUITIVO \\
\hline VISUAL & & & $\mathrm{X}$ & & & & & & & & & & VERBAL \\
\hline SECUENCIAL & & & & & & & & $\mathrm{X}$ & & & & & GLOBAL \\
\hline
\end{tabular}

En cuanto a la dimensión Sensorial - Intuitiva, un 56,6 \% de los estudiantes encuestados, muestran un equilibrio entre lo sensorial y lo intuitivo, con una preferencia moderada hacia lo sensorial (Figura 1), coincidiendo con Campos y Campos (2018), lo que favorece el eje central de la Sanidad Animal que es el diagnóstico clínico, por la necesidad del uso de los órganos de los sentidos a la hora de aplicar los métodos de exploración (inspección, palpación, percusión y auscultación), tan importante en el diagnóstico y en la recopilación de datos necesarios para poder llegar a una prognosis certera en el reconocimiento temprano de una enfermedad, y su adecuado control y tratamiento.

\section{Figura 1}


Comparación de las Dimensiones del Aprendizaje Según Inventario de Felder y Silverman (1988) Aplicado a los Estudiantes.

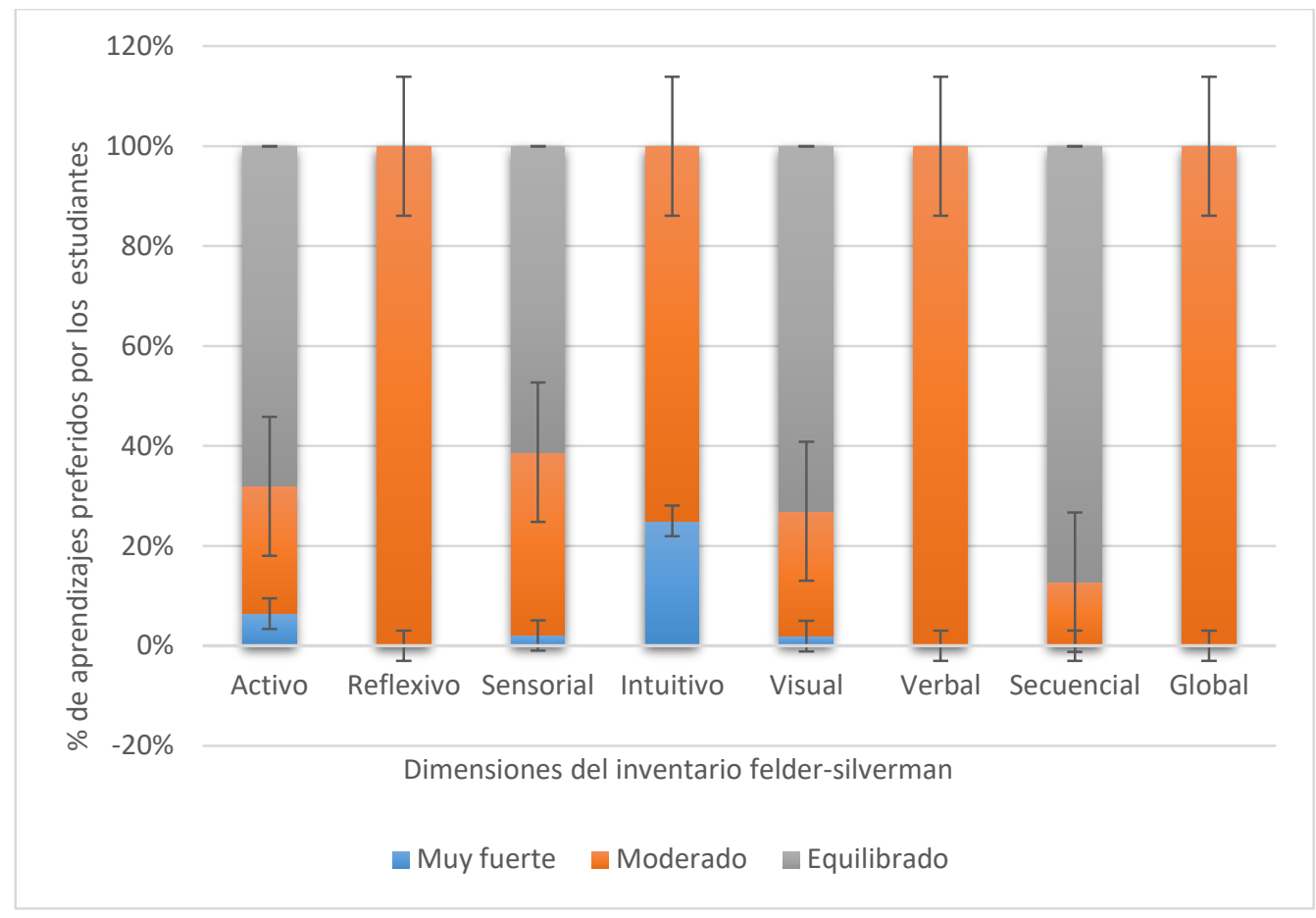

El 71, $7 \%$ de los estudiantes mostraron un equilibrio entre lo visuales y lo verbal, con preferencia moderada hacia lo visual (Figura 1), lo que se contrapone con los resultados de Gómez Ramírez et al (2021), cuando se trata de asignaturas relacionadas con la salud, tanto humana o animal, como es el caso de la presente investigación, ya que los mismos deben desarrollar destrezas y actitudes precisas, para darle una atención adecuada a los animales, en cuanto al manejo zootécnico, la prevención y curación de enfermedades, y así poder garantizar el bienestar animal que cada especie requiere.

Esta última dimensión, también es trascendental en un estudiante que recibirá Sanidad Animal, al ser imprescindible la práctica hacia la observación constante de los hatos, de los signos y síntomas clínicos como: el aspectos físicos (el color del pelaje), el movimiento de los ojos y orejas, anomalías en los aplomos, el consumos de alimentos y agua, el comportamiento colectivo de la manada, el estado de la orina y las heces fecales, los síntomas respiratorios o de cualquier otra naturaleza, que indiquen una idea precisa del estado de salud de las diferentes crianzas de animales, lo que permitirá relacionar esta capacidad visual con una mejor asimilación del proceso de aprendizaje, imprescindible a la hora del diagnóstico clínico (Losada et al, 2021; Brito-Orta y Espinosa Tanguma, 2015). 
El 77, 4\% de los estudiantes encuestados muestra equilibrio entre el aprendizaje secuencial y lo global (Figura 1), lo que indica que el docente tendrá que facilitar la resolución con métodos y procedimientos de problemas relacionados a la asignatura Sanidad Animal, que propicien la creatividad y la innovación, y que luego sean replicados por los estudiantes, de acuerdo con Arenas et al (2014), basados en el estilo propio de cada estudiante, y en la realidad objetiva que sobre la salud aparecen en las diferentes explotaciones animales. También coincide con Gutiérrez Tapias (2018), Martínez y Felicetti, (2016) y Díaz-Véliz et al, (2009), quienes confirman la tendencia mostrada en este trabajo, donde los estudiantes no modificaron significativamente sus estilos de aprendizajes en cuanto a esta dimensión.

\section{CONCLUSIONES}

La presente investigación mostró que los estilos de aprendizajes de los estudiantes encuestados, gracias al inventario de Felder-Silverman (1988), tuvieron una tendencia significativa hacia el aprendizaje activo, sensorial y visual, lo que favorece el desarrollo de la asignatura Sanidad Animal, por su gran componente teórico-práctico sustentado en beneficio de la salud de las diferentes especies productivas, además de los principios psicopedagógicos en la formación del ingeniero Agropecuario. Sin embargo, el aprendizaje secuencial mostró un equilibrio con el aprendizaje global, que aunque no es menos cierto que esto favorece la asimilación de los contenidos a recibir por los estudiantes, también indica que el docente tendrá que proporcionar métodos y estrategias didácticas para que los futuros profesionales se puedan apropiar de los mismos.

\section{REFERENCIAS BIBLIOGRAFICAS}

Alonso, C. M., Gallego, D. J., y Honey, P. (2007). Los estilos de aprendizaje. Ediciones Mensajero. $\quad 7 \mathrm{ma} \quad$ Edición. Bilbao. file:///C:/Users/Aime/Downloads/Alonso_Catalina_M_et_al_Los_Estilos_de_A $\% 20(2) . p d f$

Altuna Urdin, J., Cruz Iglesias, E., Aierbe Barandiaran, A., Madinabeitia Ezkurra, A., y Marko Juanikorena, I. (2021). Estudio de los espacios profesionales actuales de la Pedagogía: La voz del alumnado y de los profesionales. Teoría De La Educación. Revista Interuniversitaria, 33(2), 195-215. https://doi.org/10.14201/teri.23714

Arenas, E. P. (2017). Estrategias de estilos de aprendizaje de estudiantes: Proceso de validación. Revista Alteridad, 12(2). http://orcid.org/0000-0003-4689-687X 
Balduzzi, E. (2015). El cultivo de las virtudes a través de los episodios de aprendizaje situados (EAS). Teoría de la Educación. Revista Interuniversitaria, 27(1), 155167.

https://doi.org/10.14201/teoredu2015271155167

Brito-Orta, M.D. y Espinosa Tanguma, R. (2015). Evaluación de la fiabilidad del cuestionario sobre estilos de aprendizaje de Felder y Soloman en estudiantes de medicina. Investigación en Educación $\quad$ Médica., 4, 28-35. http://orcid.org/10.1016/S2007-5057(15)72166-6

Batista Casacó, A. R., Mendoza Zambrano, A., Vásconez Montufar, G. H., Torres Navarrete, Y. G., Méndez Martínez y Simón Fostén, M. (2019). Proceso formativo medio ambiental en la carrera de ingeniería zootécnica, desde la perspectiva de la sanidad animal. Universidad y Sociedad, 11(5), 287-293. https://orcid.org/0000-0002-1039-7414

Campos, M. G. y Campos, R. Estilos de aprendizaje preferidos por los estudiantes de nutrición. $\quad$ Revista UNAM, 29:2. http://dx.doi.org/10.22201/fq.18708404e.2018.2.63732

Castellanos Simons, D. (2001). Hacia una concepción del aprendizaje desarrollador. Colección Proyectos. https://www.coursehero.com/file/78619294/HaciaUnaConcepci\%C3\%B3nDelA prendizaje-Desarrolladordoc/

Díaz-Véliz, G., Mora, S., Lafuente-Sánchez, J.V., Gargiulo, P.A., Bianchi, R., Terán, C., Gorena, D. Arce, J. y Escanero-Marcen, J. F. (2009). Estilos de aprendizaje de estudiantes de medicina en universidades latinoamericanas y españolas: relación con los contextos geográficos y curriculares. Educación Médica, 12: 3.

García Gajardo, F., Fonseca Grandón, G. y Concha Gfell, L. (2015). Aprendizaje y rendimiento académico en educación superior: un estudio comparado. Revista Electrónica Actualidades Investigativas en Educación, 15 (3).

Gómez Ramírez, E., Calvo Soto, Chapal Chapal, L. Y. y García Álvarez, A. (2021). Estilos de aprendizaje de estudiantes de fisioterapia de cursos básicos y prácticas formativas de una universidad en Colombia. Educación Médica, 22, 23-27 https://doi.org/10.1016/j.edumed.2018.11.002 
Foy Valencia, P. (2017). Consideraciones jurídicas sobre la Salud Animal. Dialnet, 23, 26-49.

Gutiérrez Tapias, M. (2018). Estilos de aprendizaje, estrategias para enseñar. Su relación con el desarrollo emocional y "aprender a aprender". Tendencias Pedagógicas, 31 , 83-96. https://doi.org/10.15366/tp2018.31.004

Keefe, J. W.; Monk, John S. (1986). Eric. https://biblioteca.uoc.edu/es/Coleccion-digitalpor-areas-de-estudio/coleccion/ERIC-Educational-Resources-Information-

\section{Center/}

Leal Bolaño, N., Aguilera Cuenca, H., Egea Arciniegas, T., y Escobar Jurado, S. (2019). Estilos de Aprendizaje de Estudiantes Universitarios Principiantes con Modalidad Virtual. Cultura Educación y Sociedad, 10(2), 47-62. https://doi.org/10.17981/cultedusoc.10.2.2019.04

Losada, J. L., Hernández, E., Leiva, L. Villacís, S., Losada, J. O. (2021). A propósito del principio de visualización en la enseñanza clínica en educación. Educación Médica, 22, 172-176. https://doi.org/10.1016/j.edumed.2020.06.007

Juárez Lugo, C. S. Rodríguez Hernández, G. L. y Montijo, E. (2012). El cuestionario de estilos de aprendizaje chaea y la escala de estrategias de aprendizaje ACRA como herramienta potencial para la tutoría académica. Revista Estilos de Aprendizaje, 10(5).

Martínez, J. y Felicetti, V. L. (2016). Estilos de aprendizaje en los estudiantes de enfermería en una universidad de Colombia. Contrapontos, 16, 209-226.

OIE. (2010). Informe de la reunión de la comisión de normas sanitarias de la OIE para los animales terrestres. París. https://www.oie.int/fileadmin/Home/esp/Internationa_Standard_Setting/docs/pdf /E_TAHSC_Sept2010_Intro.pdf

Pérez Matatoros, P. G. (2021). Investigación educativa y cambio social. Octaedro, 255 pp. Teoría De La Educación. Revista Interuniversitaria, 33(2), 285-287.

Silva Sprok, A. (2018).Conceptualización de los modelos de. Revista de Estilos de Aprendizaje, 11(21).

Tapias, M. G., García Cué, J. L., y Vieira Barros, D. M. (2012). Estudio de las variables que influyen en los estilos de aprendizaje de diferentes grupos de alumnos del 
grado de magisterio de la universidad de Valladolid, España. Revista de Estilos de Aprendizaje, 5(10).

Torrano, F., Juan Luis Fuentes, J. L. y Soria, M. (2017). Aprendizaje autorregulado: estado de la cuestión y retos psicopedagógicos. Perfiles educativos, 39(156). https://doi.org/10.22201/iisue.24486167e.2017.156.58290 